\title{
EFICÁCIA DO TREINAMENTO DE HABILIDADES FONOLÓGICAS EM CRIANÇAS DE RISCO PARA DISLEXIA
}

\author{
Efficacy of phonological skills training program \\ in children under risk of dyslexia
}

\author{
Cíntia Cristina Fadini ${ }^{(1)}$, Simone Aparecida Capellini ${ }^{(2)}$
}

\begin{abstract}
RESUMO
Objetivos: identificar os sinais da dislexia em escolares de $1^{\text {a }}$ série e verificar a eficácia do programa de treinamento fonológico em crianças de risco para a dislexia que freqüentam a $1^{\text {a }}$ série escolar e investigar dentre as crianças de risco que não apresentaram melhora após o treinamento se há presença do quadro de dislexia por meio de diagnóstico interdisciplinar. Método: participaram deste estudo 30 crianças de 1 a série de ensino público, de ambos os gêneros, na faixa etária de 6 a 7 anos de idade. Neste estudo foi realizada a adaptação brasileira da pesquisa sobre treinamento de habilidades fonológicas composta de pré-testagem, intervenção e pós-testagem. Em situação de pré e pós-testagem, todas as crianças foram submetidas à aplicação do teste para a identificação precoce dos problemas de leitura e aquelas que apresentaram desempenho inferior a $51 \%$ das provas do teste foram divididas em Grupo I (GI): composto por 13 crianças submetidas ao programa de treinamento; e em Grupo II (GII): composto por 17 crianças não submetidas ao programa de treinamento. Resultados: os resultados deste estudo revelaram diferenças estatisticamente significantes, evidenciando que das 13 crianças submetidas ao programa, 11 apresentaram melhor desempenho em situação de pós-testagem em relação à pré-testagem. Apenas 2 crianças não responderam à intervenção proposta, sendo submetidas à avaliação interdisciplinar. Conclusão: a realização do programa de treinamento das habilidades fonológicas foi eficaz para as crianças de risco para dislexia, comprovados pela melhora das habilidades fonológicas e de leitura em situação de pós-testagem em relação à pré-testagem.
\end{abstract}

DESCRITORES: Dislexia; Avaliação; Reabilitação; Leitura; Criança

\section{INTRODUÇÃO}

A dislexia é uma desordem definida como uma dificuldade de realizar a leitura, mesmo com inteligência dentro dos padrões da normalidade,

(1) Fonoaudióloga; Discente do Curso de Fonoaudiologia da Faculdade de Filosofia e Ciências da Universidade Estadual Paulista - FFC/UNESP, Marília, SP; Bolsista de Iniciação Científica do Conselho Nacional de Desenvolvimento Científico e Tecnológico - CNPq.

(2) Fonoaudióloga; Docente do Departamento de Fonoaudiologia e Programa de Pós-Graduação em Educação da Faculdade de Filosofia e Ciências da Universidade Estadual Paulista - FFC/UNESP, Marília, SP; Doutora e PósDoutora em Ciências Médicas pela Faculdade de Ciências Médicas da Universidade Estadual de Campinas, FCM/ UNICAMP, Campinas, SP.

Conflito de interesses: inexistente motivação e educação adequadas. Estima-se que a prevalência da dislexia está entre 5 a $17 \%$ da população escolar, sendo a mesma caracterizada pela dificuldade no processamento da linguagem, primeiramente no processamento fonológico da fala e posteriormente no processamento fonológico envolvido na leitura1.

Os sinais da dislexia podem ser evidenciados durante o período escolar e, segundo autores ${ }^{2-5}$, estes sinais se referem à: fala ininteligível; imaturidade fonológica; redução de léxico; dificuldade em aprender o nome das letras ou os sons do alfabeto; dificuldade para entender instruções, compreender a fala ou material lido; dificuldade para lembrar números e palavras em sequência; dificuldade para lembrar sentenças ou estórias; ao atraso de fala; confusão direita-esquerda, embaixo, em cima, frente-atrás (palavras-conceitos); dificuldade em 
processar sons das palavras e história familial positiva de problemas de fala, linguagem e desenvolvimento da leitura.

Assim, é preciso que tanto professores como fonoaudiólogos estejam atentos ao desenvolvimento da linguagem expressiva e receptiva das crianças no início da alfabetização para que a identificação e a detecção dos sinais da dislexia possam ocorrer precocemente e os problemas acadêmicos decorrentes de alterações cognitivo-linguísticas minimizadas por meio da realização de programas de treinamento fonológico.

Estudos internacionais apontam para a necessidade de realização de identificação dos problemas de aprendizagem em início da escolarização e conseqüente intervenção precoce em crianças que em séries iniciais apresentem desempenho abaixo do esperado se comparado ao seu grupo-classe nos fatores preditivos para o bom desempenho em leitura como: conhecimento do alfabeto, nomeação automática rápida, repetição de não-palavras e habilidades de consciência fonológica. Estas crianças são denominadas na literatura internacional como crianças de risco para a dislexia ${ }^{5-10}$.

O trabalho precoce com crianças em início da alfabetização passa a ser um importante mecanismo de auxílio para a identificação e o diagnóstico da dislexia, pois a existência do risco não necessariamente significa a presença de um quadro de dislexia que significa necessariamente a presença de uma condição determinada genética e neurologicamente ${ }^{11,12}$.

Desse modo, os programas de treinamento com a base fonológica têm por objetivo verificar se depois da realização de programas específicos enfocando as habilidades fonológicas e cognitivas alteradas, as crianças apresentam melhora na aprendizagem da leitura ou se permanecem com as defasagens nessas habilidades, o que significa que as crianças apresentam realmente uma desordem de origem genético-neurológica que compromete a aquisição e desenvolvimento de habilidades perceptivas e linguísticas e que por isso devem ser submetidas a avaliações interdisciplinares para a investigação do quadro de dislexia ${ }^{2,5,12,13}$.

Assim, a importância para a prática clínica e educacional desses estudos consiste em que a falta de resposta ao treinamento fonológico precoce com os sinais da dislexia pode ser considerada um critério para o diagnóstico da dislexia do desenvolvimento ${ }^{12-14}$.

Em decorrência do exposto, este estudo tem por objetivo identificar os sinais da dislexia em escolares de $1^{\text {a }}$ série e verificar a eficácia do programa de treinamento fonológico em crianças de risco para a dislexia que freqüentam a $1^{\text {a }}$ série escolar e investigar dentre as crianças de risco que não apresentaram melhora após o treinamento se há presença do quadro de dislexia por meio de diagnóstico interdisciplinar.

\section{MÉTODO}

Este estudo se caracteriza por ser experimental e transversal realizado com crianças de ensino público municipal da cidade de Marília-SP.

Participaram deste estudo 30 crianças de $1^{\text {a }}$ série do ensino público fundamental municipal, de ambos os gêneros e na faixa etária entre 6 anos e 11 meses a 7 anos e 3 meses, nível sócio-econômico médio-inferior (Tabela 1), submetidos a metodologia de alfabetização com enfoque silábico e de leitura de textos. Todas as crianças freqüentaram a educação infantil anteriormente ao início da primeira série escolar e pertenciam à mesma sala de aula.

TABELA 1 - Distribuição das crianças da 1aㅗ série submetidas à avaliação para a identificação dos sinais de dislexia segundo gênero

\begin{tabular}{ccccc}
\hline Grupos & Masculino & \multicolumn{3}{c}{ Feminino } \\
\hline & $\mathbf{N}$ & $\%$ & $\mathbf{N}$ & $\%$ \\
I & 06 & $(20 \%)$ & 07 & $(23 \%)$ \\
II & 11 & $(37 \%)$ & 06 & $(20 \%)$ \\
\hline
\end{tabular}

As crianças que participaram deste estudo não apresentaram anotações referentes à deficiência mental, física, sensorial ou múltipla em prontuário escolar, sendo isto considerado o critério de inclusão das crianças neste estudo. Como critério de exclusão foi considerado anotações referentes à deficiência mental, física, sensorial ou múltipla em prontuário escolar e a falta de assinatura dos pais ou responsáveis no termo de consentimento livre e esclarecido. 
A identificação do risco para dislexia nas crianças deste estudo foi realizada a partir da aplicação de provas de avaliação elaboradas a partir de descrição em pesquisa sobre treinamento de habilidades fonológicas e conhecimento de letrasom utilizadas para levantamento dos sinais da dislexia $^{6}$.

Inicialmente todas as crianças deste estudo foram submetidas aos seguintes procedimentos:

- Teste para identificação precoce dos problemas de leitura ${ }^{6}$ : Foi realizada a elaboração de 7 provas para identificação precoce dos problemas de leitura baseadas na descrição da pesquisa original $^{6}$ que ofereceu suporte teórico para a realização deste estudo. As provas que compõem o Teste estão descritas a seguir e foram normalizadas para população de escolares brasileiros em estudo anterior ${ }^{15}$ :

1. Conhecimento do alfabeto: foi apresentado para a criança o alfabeto para que identificasse o nome da letra e o valor sonoro de cada letra apresentada.

2. Consciência fonológica: composta por subtestes de produção de rima, identificação de rima, segmentação silábica, produção de palavras a partir do fonema dado, síntese fonêmica, análise fonêmica, identificação de som inicial.

2.1. Produção de rima: foram apresentadas para a criança 20 palavras auditivamente e solicitado que dissesse uma palavra que terminasse com o mesmo som.

2.2. Identificação de rima: foram apresentados para a criança 20 grupos de três em três palavras auditivamente e solicitado que identificasse as palavras que terminassem com o mesmo som.

2.3. Segmentação silábica: foram apresentadas para a criança 21 palavras auditivamente (dissílabas, trissílabas e quadrissílabas) e solicitado que separasse as palavras por silabas.

2.4. Produção de palavras a partir do fonema dado: foram apresentados para a criança os sons do alfabeto $e$ solicitado que dissesse uma palavra que começasse com o mesmo som.

2.5. Síntese fonêmica: foram apresentadas para a criança 21 palavras auditivamente separadas por sons e solicitado que dissesse a palavra formada.

2.6. Análise fonêmica: foram apresentadas para a criança 21 palavras auditivamente e solicitado que dissesse os sons de cada letra das palavras apresentadas.

2.7. Identificação de som inicial: foram apresentadas para a criança 21 palavras auditivamente e solicitado que dissesse o som inicial da primeira letra de cada palavra apresentada.

3. Memória de trabalho: foram apresentadas para a criança 24 pseudopalavras auditivamente e solicitado que repetisse como havia entendido.

4. Velocidade de acesso à informação fonológica (nomeação rápida): foram apresentadas para a criança 7 sequências intercaladas de desenhos coloridos (carro, bola, pato, casa e chave) e solicitado que realizasse a nomeação rápida.

5. Atenção visual: foram apresentadas para a criança 10 figuras coloridas e solicitado que identificasse entre duas palavras a que correspondia às figuras.

6. Leitura de palavras e pseudopalavras: foram apresentadas para a criança 40 palavras visualmente (20 palavras e 20 pseudopalavras) e solicitado que realizasse a leitura em voz alta.

7. Compreensão de frases a partir de figuras apresentadas: foram apresentadas para a criança 20 frases incompletas com figuras ilustrativas e solicitado que observasse as figuras e completasse as frases.

A aplicação deste procedimento durou em média 50 minutos e foi realizado individualmente em uma única sessão durante o horário de aula das crianças, com a anuência e autorização da professora e da direção da escola em que foi realizado o estudo para retirada das crianças da sala de aula para aplicação do procedimento em local apropriado, cedido pela escola.

O material linguístico utilizado para a elaboração das provas descritas foi retirado de um banco de palavras de livros didáticos de $1^{\underline{a}}$ a $4^{\underline{a}}$ séries, utilizados na rede municipal de ensino de Marília-SP. As palavras utilizadas para a elaboração das provas seguem as regras de decodificação do português brasileiro tanto para palavras como para pseudopalavras: a) regra de correspondência grafofonêmica independente do contexto e b) regra de correspondência grafofonêmica dependente do contexto ${ }^{(16)}$. Entretanto, levou-se em consideração para a elaboração das provas, a seleção de palavras de alta freqüência presentes em todos os livros de $1^{\text {a }}$ a $4^{a}$ séries, para que o estímulo linguístico não interferisse no desempenho da criança. 
Dentre as 30 crianças (100\%) submetidas ao procedimento de identificação dos sinais da dislexia, 13 crianças (43\%) apresentaram desempenho inferior, conforme critério descrito anteriormente, compondo o grupo submetido ao treinamento. A partir desse achado as crianças foram divididas em dois grupos:

- Grupo I (GI): composto por 13 crianças com desenvolvimento típico que apresentaram risco para dislexia, submetidas ao programa de treinamento das habilidades fonológicas, sendo $46 \%$ do gênero masculino e 54\% do gênero feminino.

- Grupo II (GII): composto por 17 crianças sem risco para dislexia, não submetidas ao programa de treinamento das habilidades fonológicas, sendo $65 \%$ do gênero masculino e $35 \%$ do gênero feminino.

A partir da distribuição das crianças em dois grupos foi realizada a aplicação do procedimento de intervenção com as crianças distribuídas em duplas e trios. Tal como a avaliação utilizada anteriormente neste estudo, foram elaboradas estratégias para o programa de treinamento das habilidades fonológicas a partir da descrição da pesquisa original ${ }^{(6)}$, com base em critérios psicolingüísticos de adaptação para a Língua Portuguesa.

O programa de treinamento das habilidades fonológicas utilizado neste estudo foi composto por dez atividades trabalhadas em 18 sessões de 50 minutos de duração realizadas em duas sessões semanais na escola de origem das crianças, com duração total de 10 semanas. As atividades desenvolvidas no programa de treinamento estão descritas a seguir:

1. Reconhecimento do alfabeto fonêmico: Foi apresentado o alfabeto para as crianças identificarem o nome da letra e o valor sonoro de cada letra apresentada.

2. Identificação de palavras dentro de uma frase: Foram apresentadas 7 frases afirmativas auditivamente para que as crianças dividissem a frase em palavras, marcando-as por palmas.

3. Identificação e manipulação de sílabas na palavra: Foram apresentadas 2 palavras auditivamente para a identificação de sílabas iguais e, em seguida, solicitado que a criança manipulasse a segmentação silábica para a formação de novas palavras em posição inicial, medial e final. Em cada sessão foram fornecidas 6 palavras;

4. Síntese fonêmica: Foram apresentadas 7 palavras auditivamente separadas por sons para que as crianças identificassem as palavras.

5. Rima: Foram solicitadas para as crianças as palavras que terminassem com o mesmo som e a identificação de figuras que rimassem em sua nomeação.

6. Identificação e discriminação de fonemas: Foi apresentado auditivamente um fonema e solicitado que as crianças mencionassem uma palavra que começasse com este som. Depois, foram apresentadas oralmente 7 palavras e questionado se havia o fonema alvo naquelas palavras. Os fonemas foram apresentados seguindo a ordem de aquisição e desenvolvimento do sistema fonológico

7. Segmentação de fonemas: Foi apresentada para as crianças 1 palavra oralmente e solicitado que dissessem todos os fonemas desta. Nesta fase do programa foram utilizadas fichas coloridas para auxiliar as crianças a segmentar os sons das palavras auditivamente apresentadas. Em cada sessão foram fornecidas 7 palavras;

8. Subtração de fonemas: foram apresentadas para as crianças 6 palavras para que retirassem o fonema final e, em seguida, foram apresentadas 6 palavras para que retirassem o fonema inicial.

9. Substituição de fonemas: foi apresentada para as crianças 1 palavra oralmente e solicitado que retirassem o fonema inicial e o substituísse por outro, formando, assim, uma nova palavra. Em cada sessão foram fornecidas 7 palavras;

10. Transposição de fonemas: foi solicitado para as crianças que falassem os fonemas das palavras em ordem inversa da apresentada como o objetivo de formar novas palavras. Em cada sessão foram fornecidas 6 palavras;

Após o término do treinamento das habilidades fonológicas, as crianças do Gl e Gll foram submetidas novamente à aplicação do Teste para identificação precoce dos problemas de leitura a fim de verificar se os sinais da dislexia identificados no Gl se extinguiram ou se permaneceram presentes nesta população. Levando em consideração que as crianças do GII não foram submetidas ao programa de treinamento, mas continuaram em situação de aprendizagem escolar, as mesmas foram submetidas a nova aplicação do procedimento de avaliação, justamente para verificar a eficácia do treinamento e da escolarização nas crianças do GI.

Ressalta-se que, em atenção aos aspectos éticos em pesquisa com seres humanos, as crianças de risco para dislexia identificadas neste 
estudo e submetidas ao programa de treinamento, quando finalizado o tempo previsto para o treinamento, foram encaminhadas para diagnóstico interdisciplinar e tratamento fonoaudiológico no Centro de Estudos da Educação e Saúde - CEES/FFC/ UNESP se as habilidades avaliadas na primeira testagem não tivessem melhorado em situação de pós-testagem.

Este estudo foi aprovado pelo Comitê de Ética em Pesquisa da Faculdade de Filosofia e Ciências da Universidade Estadual Paulista - CEP/FFC/ UNESP sob o protocolo de $n^{\circ}=3348 / 2008$.

Para a análise estatística foi utilizado o Teste de Mann-Whitney com o objetivo de verificar possíveis diferenças entre grupos considerados em situação de pré-testagem, treinamento e pós-testagem.
Outro método de análise estatística utilizado foi à aplicação do Teste dos Postos Sinalizados de Wilcoxon com o objetivo de verificar possíveis diferenças entre os dois momentos, pré e pós-testagem, considerados na avaliação do grupo. O nível de significância adotado para a aplicação dos testes estatísticos foi de $5 \%(0,050)$. A análise dos dados foi realizada utilizando o programa SPSS (Statistical Package for Social Sciences), em sua versão 13.0.

\section{RESULTADOS}

A caracterização do desempenho das 30 crianças deste estudo no Teste para identificação precoce dos problemas de leitura está descrita na tabela 2.

Tabela 2 - Distribuição da média, desvio padrão, valor mínimo e valor máximo do desempenho obtido pelas crianças em cada prova do teste para identificação precoce dos problemas de leitura

\begin{tabular}{ccccc}
\hline Provas & Média & Desvio padrão & Mínimo & Máximo \\
\hline CA & 19,33 & 3,26 & 10,00 & 23,00 \\
PR & 5,83 & 5,84 & 0,00 & 18,00 \\
IR & 11,03 & 7,07 & 0,00 & 21,00 \\
SS & 19,47 & 2,43 & 12,00 & 21,00 \\
PP & 16,13 & 4,94 & 5,00 & 21,00 \\
SF & 0,40 & 1,54 & 0,00 & 7,00 \\
AF & 1,83 & 2,60 & 0,00 & 11,00 \\
ISI & 4,73 & 7,15 & 0,00 & 21,00 \\
MT & 19,90 & 3,17 & 13,00 & 25,00 \\
NR & 38,77 & 5,33 & 30,00 & 50,00 \\
AV & 9,60 & 0,67 & 8,00 & 10,00 \\
L & 22,90 & 14,82 & 0,00 & 40,00 \\
CF & 16,03 & 5,22 & 0,00 & 20,00 \\
\hline
\end{tabular}

Legenda: CA: conhecimento do alfabeto, PR: produção de rima, IR: identificação de rima, SS: segmentação silábica, PP: produção de palavras a partir do fonema dado, SF: síntese fonêmica, AF: análise fonêmica; ISI: identificação de som inicial, MT: memória de trabalho; NR: nomeação rápida; AV: atenção visual, L: leitura, CF: compreensão de frases.

Os dados descritos na tabela 2 apresentam os resultados de todos os participantes deste estudo, conforme demonstrado na apresentação dos dados após tratamento estatístico. Isto se fez necessário, pois as crianças que apresentaram desempenho inferior a $51 \%$ do valor máximo em pelo menos 4 provas do teste para identificação precoce dos problemas de leitura em relação (conhecimento do alfabeto, consciência fonológica, nomeação rápida e leitura de palavras e pseudopalavras) em relação ao grupo-classe foram consideradas de risco para dislexia. Assim, a tabela 2 apresenta a média e o valor máximo que foram considerados como ponte de corte para inclusão dos escolares de risco para a dislexia.

A tabela 3 apresenta a distribuição da média, desvio padrão e valor de $p$ do desempenho obtido do Gl e Gll no Teste para a identificação precoce dos problemas de leitura em situação de pré e pós-testagem. Comparando os dois momentos de 
avaliação por meio do Teste dos Postos Sinalizados de Wilcoxon, pode-se observar que as crianças do Gl submetidas ao programa de intervenção apresentaram diferença estatisticamente significante na maioria dos subtestes, com exceção de atenção visual (AV), demonstrando que o programa foi eficaz para o domínio de habilidades fonológicas e não de habilidades visuais, isto pose ser explicado pelo fato do programa enfocar explicitamente habilidades auditivas e não visuais.

Tabela 3 - Distribuição da média, desvio padrão e valor de $p$ do desempenho obtido das crianças do GI e GIl em situação de pré e pós-testagem

\begin{tabular}{|c|c|c|c|c|c|c|c|}
\hline GI & Média & $\begin{array}{l}\text { Desvio } \\
\text { padrão }\end{array}$ & Valor de $p$ & GII & Média & $\begin{array}{l}\text { Desvio } \\
\text { padrão }\end{array}$ & Valor de $p$ \\
\hline CA_pré & 17,15 & 3,76 & \multirow{2}{*}{$0,002^{*}$} & CA_pré & 21,00 & 1,37 & \multirow{2}{*}{$0,049^{*}$} \\
\hline CA_pós & 21,85 & 1,14 & & CA_pós & 21,71 & 2,87 & \\
\hline PR_pré & 2,31 & 3,50 & \multirow{2}{*}{$0,002^{*}$} & PR_pré & 8,53 & 5,91 & \multirow{2}{*}{$0,016^{\star}$} \\
\hline PR_pós & 12,15 & 5,80 & & PR_pós & 12,12 & 4,23 & \\
\hline IR_pré & 6,62 & 7,25 & \multirow{2}{*}{$0,002^{*}$} & I_R_pré & 14,41 & 4,82 & \multirow{2}{*}{$0,003^{*}$} \\
\hline IR_pós & 14,85 & 3,76 & & IR_pós & 17,47 & 2,85 & \\
\hline SS_pré & 18,31 & 2,95 & \multirow{2}{*}{$0,011^{*}$} & SS_pré & 20,35 & 1,50 & \multirow{2}{*}{0,131} \\
\hline SS_pós & 20,62 & 0,87 & & SS_pós & 20,94 & 0,24 & \\
\hline PPpré & 14,00 & 5,46 & \multirow{2}{*}{$0,002^{*}$} & PPpré & 17,76 & 3,91 & \multirow{2}{*}{0,673} \\
\hline PP_pós & 20,23 & 1,24 & & PP_pós & 18,35 & 2,34 & \\
\hline SF_pré & 0,92 & 2,29 & \multirow{2}{*}{$0,001^{*}$} & SF_pré & 0,00 & 0,00 & \multirow{2}{*}{$0,001^{*}$} \\
\hline SF_pós & 11,77 & 6,75 & & SF_pós & 5,06 & 6,04 & \\
\hline AF_pré & 0,77 & 1,54 & \multirow{2}{*}{$0,001^{*}$} & AF_pré & 2,65 & 2,98 & \multirow{2}{*}{0,413} \\
\hline AF_pós & 13,92 & 6,91 & & AF_pós & 4,47 & 7,06 & \\
\hline ISI_pré & 0,54 & 1,66 & \multirow{2}{*}{$0,001^{*}$} & ISI_pré & 7,94 & 8,08 & \multirow{2}{*}{0,754} \\
\hline ISI_pós & 20,31 & 1,03 & & ISI_pós & 7,24 & 6,59 & \\
\hline MT_pré & 18,15 & 3,08 & \multirow{2}{*}{$0,003^{*}$} & MT_pré & 21,24 & 2,59 & \multirow{2}{*}{0,593} \\
\hline MT_pós & 20,69 & 2,50 & & MT_pós & 21,24 & 2,14 & \\
\hline AV_pré & 9,31 & 0,75 & \multirow{2}{*}{0,279} & 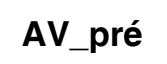 & 9,82 & 0,53 & \multirow{2}{*}{0,180} \\
\hline AV_pós & 9,62 & 0,87 & & AV_pós & 10,00 & 0,00 & \\
\hline L_pré & 9,92 & 12,20 & \multirow{2}{*}{$0,001^{*}$} & L_pré & 32,82 & 6,73 & \multirow{2}{*}{$0,012^{*}$} \\
\hline L_pós & 24,23 & 12,10 & & L_pós & 34,88 & 6,59 & \\
\hline CF_pré & 14,85 & 5,60 & \multirow{2}{*}{$0,035^{\star}$} & CF_pré & 16,94 & 4,89 & \multirow{2}{*}{0,174} \\
\hline CF_pós & 18,23 & 1,17 & & CF_pós & 18,59 & 1,37 & \\
\hline
\end{tabular}

Legenda: CA: conhecimento do alfabeto, PR: produção de rima, IR: identificação de rima, SS: segmentação silábica, PP: produção de palavras a partir do fonema dado, SF: síntese fonêmica, AF: análise fonêmica; ISI: identificação de som inicial, NR: nomeação rápida, AV: atenção visual, L: leitura, CF: compreensão de frases; Pré: Pré-testagem; Pós: Pós-testagem.

As crianças do Gl submetidas ao treinamento fonológico quanto ao teste de identificação precoce dos problemas de leitura em situação de pré-testagem obtiveram desempenho abaixo do esperado em todas as atividades propostas, enquanto que o Gll nessa mesma situação obteve o desempenho esperado, exceto nas atividades de Síntese fonêmica (SF), Análise fonêmica (AF), Identificação de som inicial (ISI).

$\mathrm{Na}$ situação de pós-testagem, as crianças do GI e Gll obtiveram desempenho próximo, exceto em atividades como: Conhecimento do alfabeto (CA), 
Produção de Rima (PR), Produção de palavras a partir do fonema dado (PP), Síntese fonêmica (SF), análise fonêmica $(A F)$, identificação de som inicial (ISI).

Entretanto, entre as crianças do GIl, evidenciou-se diferença estatisticamente significante nos subtestes de conhecimento do alfabeto (CA), produção de rima (PR), identificação de rima (IR), segmentação fonêmica (SF) e leitura de palavras (L), demonstrando que o conteúdo oferecido pelo professor em sala de aula favoreceu a melhora do desempenho destas crianças nos subtestes descritos anteriormente (tabela 3 ). Isto mostrou que as crianças submetidas ao programa de treinamento fonológico se beneficiaram tanto das estratégias de base fonológica do programa como das atividades silábicas desenvolvidas pelo professor em sala de aula, evidenciando uma relação de causa-efeito necessária para a alfabetização de um sistema de escrita com base alfabética como o português.

$\mathrm{Na}$ tabela 4, pode-se observar que as crianças do Gl em situação de pré-testagem apresentaram maior tempo de nomeação rápida de figuras do que o GII, e que este tempo diminuiu na pós-testagem, aproximando-se do tempo de nomeação realizado pelas crianças do Gll. Apesar de não haver diferença estatisticamente significante, esse dado demonstra que o programa favoreceu a fusão rápida de estímulos em sucessão, exigida tanto em tarefas de nomeação rápida como em leitura.

Tabela 4 - Distribuição da média, desvio padrão, valor mínimo, valor máximo e valor de $p$ do desempenho das crianças do GI e Gll em situação de pré e pós-testagem no subteste de nomeação rápida de figuras (NR)

\begin{tabular}{ccccccc}
\hline & $\begin{array}{c}\text { Par de } \\
\text { Variáveis }\end{array}$ & Média & Desvio padrão & Mínimo & Máximo & Valor de $\mathbf{p}$ \\
\hline $\bar{\sigma}$ & NR_pré & 38,77 & 5,33 & 30,00 & 50,00 & 0,093 \\
& NR_pós & 35,38 & 5,90 & 27,00 & 47,00 & \\
$\overline{\bar{\sigma}}$ & NR_pré & 34,82 & 6,37 & 25,00 & 50,00 & 0,492 \\
\hline
\end{tabular}

Legenda: NR: Nomeação Rápida; Pré: Pré-testagem; Pós: Pós-testagem.

Dentre as 13 crianças (100\%) submetidas ao programa de treinamento de habilidades fonológicas, apenas $2(15 \%)$ continuaram apresentando desempenho inferior a $51 \%$ do valor máximo em pelo menos 4 provas do teste para identificação precoce dos problemas de leitura em relação (conhecimento do alfabeto, consciência fonológica, nomeação rápida e leitura de palavras e pseudopalavras). Essas crianças foram encaminhadas para uma avaliação interdisciplinar composta por avaliação neurológica e neuropsicológica no Centro de Estudos da Educação e Saúde - CEES/FFC/ UNESP, tendo sido verificado em uma criança o quadro de atraso cognitivo limítrofe e em outra 0 quadro de dislexia. Depois do diagnóstico interdisciplinar, essas crianças permaneceram no CEES para início de intervenção fonoaudiológica.

\section{DISCUSSÃO}

Os resultados deste estudo revelaram que as 11 crianças consideradas de risco para a dislexia depois de serem submetidas ao programa de treinamento fonológico melhoraram o desempenho em estratégias de percepção, discriminação, armazenamento e recuperação de informação fonêmica, conforme descrito em estudos internacionais ${ }^{2,5,6,12}$.

Em decorrência disso, essas crianças apresentaram melhora nas habilidades cognitivolinguísticas, visto que um bom desempenho em percepção, discriminação e tempo de armazenamento da informação linguística provoca a melhora no uso de habilidades de leitura e compreensão, conforme descrito na literatura ${ }^{6}$.

O programa de treinamento adaptado neste estudo enfocou a atenção, discriminação e memória auditiva de forma direta em todas as sessões, proporcionando às crianças uma melhor e maior atenção à base fonológica do sistema de escrita da Língua Portuguesa. Assim, tanto as crianças com sinais de dislexia como as crianças sem estes sinais apresentaram melhora nas habilidades fonológicas, apontando para a necessidade de uma reflexão sobre a metodologia de alfabetização utilizada em situação de sala de aula, uma vez que as 
habilidades de atenção, discriminação e percepção dos sons trabalhadas neste programa proporcionaram um aumento na sensibilidade e percepção fonológica, refletindo também na melhora do tempo de realização da leitura. Esses achados foram descritos também por autores internacionais em seus estudos 2, 5, 6,12-14.

Entretanto, pode-se destacar que depois do programa de treinamento fonológico das 13 crianças consideradas de risco para a dislexia, apenas 2 crianças não apresentaram melhora no desempenho em situação de pós-testagem. Portanto, 11 crianças apresentaram apenas falhas no processo de alfabetização e não o quadro de dislexia, isto quer dizer que quando a metodologia de alfabetização não oferece instrução formal sobre o mecanismo de conversão fonema-grafema, problemas em habilidades consideradas importantes para aprendizagem da leitura em um sistema de escrita com base alfabética pode ocorrer, gerando características da dislexia em escolares em fase de alfabetização. ${ }^{(5-7)}$

Apenas 1 escolar deste estudo que não respondeu ao treinamento fonológico, apresentou atraso cognitivo limítrofe em avaliação neuropsicológica, isto nos leva a considerar duas hipóteses, a primeira se refere a importância de levarmos em consideração não apenas a testagem neuropsicológica realizada, que embora seja fundamental, a interpretação dos seus resultados deve levar em conta o contexto étnico e cultural, o nível educacional e a motivação que podem gerar desempenho artificialmente baixo em alguns subtestes, refletindo em um quoeficiente intelectual rebaixado, e a segunda, o fato do rebaixamento leve ou limítrofe ser detectado em escolares em idade e seriação mais avançadas, quando o escolar começa a falhar em decorrência de maior exigência de armazenamento e recuperação de memória, resultando em fracasso escolar decorrente do aumento de conteúdo escolar, descrito na literatura ${ }^{17,18}$.

Os achados deste estudo evidenciam que os sinais descritos na literatura nacional e internacional $^{2-5}$ sofrem interferência direta da metodologia de ensino, o que justifica o fato de os fonoaudiólogos juntamente com os professores utilizarem programas de intervenção com base fonológica para identificar e intervir precocemente nos sinais da dislexia e, dessa forma, diminuir o número de encaminhamentos de crianças com dificuldades de aprendizagem de origem pedagógica para a realização de diagnóstico fonoaudiológico. Essa prática corrobora estudos descritos na literatura ${ }^{11-21}$.

Assim, os achados deste estudo apresentam o treinamento da habilidade fonológica em crianças de $1^{a}$ série com risco para a dislexia como um instrumento de auxílio ao diagnóstico de uma condição determinada genética e neurologicamente, como a dislexia, uma vez que, conforme descrição na literatura internacional ${ }^{6-10}$, o fracasso da criança de risco a este tipo de treinamento é um critério diagnóstico para a dislexia, isto ocorreu com apenas 1 crianças deste estudo, que após não responder ao treinamento proposto, foi identificada a dislexia em situação de avaliação interdisciplinar.

Estes achados apontam para o fato de que quanto menor for a instrução na base fonológica para a aprendizagem da leitura, maior será o risco de os fonoaudiólogos identificarem erroneamente uma criança como sendo disléxica.

\section{CONCLUSÃO}

Neste estudo foram identificadas 13 crianças com sinais de risco para a dislexia, sendo que após o programa de intervenção, apenas 2 crianças não apresentaram melhora nas habilidades cognitivolinguísticas treinadas em situação de intervenção. Assim, podemos considerar que a realização do programa de treinamento das habilidades fonológicas foi eficaz para as crianças de risco para dislexia, comprovada pela melhora das habilidades fonológicas e de leitura em situação de póstestagem em relação à pré-testagem na maioria das crianças deste estudo.

O programa realizado neste estudo possibilitou verificar que a criança que não respondeu ao programa de intervenção confirmou o diagnóstico da dislexia evidenciando que a falta de resposta a um programa de intervenção estruturado é um indicativo da presença de um quadro determinado genética e neurologicamente como a dislexia.

\section{AGRADECIMENTOS}

Ao CNPq pela concessão de bolsa de iniciação científica da primeira autora e da bolsa produtividade em pesquisa da segunda autora. 


\begin{abstract}
Purpose: to identify the symptoms of dyslexia in $1^{\text {st }}$ grade students and verify the effectiveness of the phonological training program in children under risk of dyslexia and verify children under risk who have not improved after training for the presence of dyslexia through interdisciplinary diagnosis. Method: the participants of this study were 30 children from the 1st grade in public schools, of both genders, from 6 to 7 years and 11 months old. In this study we adapted the research about training phonological skills. The phonological training program was adapted in three stages: pre-testing, training and posttesting. All children were submitted to the test for early identification of reading problems; only 13 children showed difficulty in performing more than $51 \%$ of the test and were submitted to the training program. The children were divided as follows: Group I (GI) with 13 children who were submitted to the program; and group II (GI) with 17 children who were not submitted to the program. Results: there were statistically significant differences, suggesting that 11 children submitted to the program showed better performance at a post-test when compared to pre-testing, only 2 children did not respond to the proposed intervention, being submitted to an interdisciplinary evaluation. Conclusions: the accomplishment of the phonological skills training program was effective for the children under risk of dyslexia, proven for the improvement of the phonological and reading skills in a situation of posttesting in relation to pre-testing.
\end{abstract}

KEYWORDS: Dyslexia; Evaluation; Rehabilitation; Reading; Child

\section{REFERÊNCIAS}

1. Temple E, Poldrack RA, Protopapas S, Nagarajan S, Salz T, Tallal P, et al. Disruption of neural response to rapid acoustic stimuli in dyslexia: evidence from functional MRI. Proc Natl. Acad. Sci USA. 2000; 97(25):13907-12.

2. Hindson B, Byrne B, Fielding-Barnsley RF, Newman C, Hine DW. Assessment and early instruction of preschool children at risk for reading disability. J. Educ. Psyc. 2005; 97(4):687-704.

3. Harrison, A. G., Edwards, M. J. and Parker, K. C. H. (2008), Identifying students feigning dyslexia: preliminary findings and strategies for detection. Dyslexia. 2008; 14(3):228-46.

4. Lobier M, Valdois S. Developmental dyslexia and intervention methods: assessment criteria. Neuropsychol. 2009; 1(2):102-9.

5. López-Escribano C, Beltrán JA. Early predictors of reading in three groups of native Spanish speakers: Spaniards, Gypsies, and Latin Americans. Span J Psychol. 2009; 12:84-95.

6. Schneider W, Roth E, Ennemoser M. Training phonological skills and letter knowledge in children at risk for dyslexia: a comparison of three kindergarten intervention programs. J. Educ. Psy. 2000; 92(2):284-95.

7. Regtvoort AGFM, Van Der Leij A. Early intervention with children of dyslexic parents: effects of computer-based reading instruction at home on literacy acquisition. Learn Individ Differ. 2007; 17(1):35-53.

8. Vaughn $S$, Linan-Thompson $S$, Mathes PG, Cirino PT, Carlson CD, Pollard-Durodola SD, et al. Effectiveness of Spanish intervention for firstgrade English language learners at risk for reading difficulties. J. Learn. Disab. 2006; 39(1):56-73.

9. Landry SH, Swank PR, Smith KE, Assel MA, Gunnewig SB. Enhancing early literacy skills for preschool children bringing professional development model to scale. J. Learn. Disab. 2006; 39(4):306-24 .

10. Gijsel MAR, Bosman AMT, Verhoeven L. Kindergaten risk factors, cognitive factors, and teacher judgments as predictors of early reading in dutch. J. Learn. Disab. 2006; 39(6):558-71.

11. Simmons DC, Kame'enui EJ, Harn B, Coyne MD, Stoolmiller, M Santoro, LE, et al. Attributes of effective and efficient kindergarten reading intervention: An examination of instructional time and design of instruction specificity. J. Learn. Disab. 2007; 40:331-47.

12. Harn BA, Linan-Thompson S, Roberts G. Intensifying instruction: does additional instructional time make a difference for the most at-risk first graders?. J. Learn Disab. 2008; 41(2):115-25.

13. Simmons DC, Coyne MD, McDonagh S, Harn, BA, Kame'enui, EJ. Indexing response to intervention-A longitudinal study of reading risk from kindergarten through third grade. ?. J. Learn Disab. 2008; 41(2):158-73. 
14 López-Escribano C. Contribuciones de la neurociencia al diagnóstico y tratamiento educativo de la dislexia del desarrollo. Rev Neurol. 2007; 44(3):173-80.

15. Capellini SA, Sampaio MN, Matsuzawa MT, Oliveira AM, Fadini CC, Martins, MA. Protocolo de identificação precoce dos problemas de leitura: estudo preliminar com escolares de 1 을 ano escolar. Psicopedagogia. 2009; 81:367-75.

16. Scliar-Cabral L. Princípios do sistema alfabético do português do Brasil. São Paulo: Contexto; 2003. 17. Rauch A, Hoyer J, Guth S, Zweier C, Kraus $C$, Becker $C$, et at. Diagnostic yield of various genetic approaches in patients with unexplained developmental delay or mental retardation. Am J Med Genet. 2006; 140A:2063-74.
18. Battaglia A, Carey JC. Diagnostic evaluation of developmental delay/mental retardation: an overview. Am J Med Genet. 2003; 117C:3-14.

19. Salgado CA, Capellini SA. Programa de remediação fonológica em escolares com dislexia do desenvolvimento. Pró-Fono R. Atual. Cient. 2008; 20:31-6.

20. Calhoon MB. Effects of a peer-mediated phonological skill and reading comprehension program on reading skill acquisition for middle school students with reading disabilities. J. Learn. Disab. 2005; 38(5):424-33.

21. Tressoldi PE, Vio C, lozzino R. Efficacy of an intervention to improve fluency in children with development dyslexia in a regular ortography. J. Learn. Disab. 2006; 40(3): 203-9.

Endereço para correspondência:

Simone Aparecida Capellini

Rua Hygino Muzzy Filho, 737,

Campus Universitário

Marília - SP

CEP: $17525-900$

E-mail: sacap@uol.com.br 